\title{
Design of a high and low pair fusion lower limb exoskeleton robot
}

\author{
Shufeng Tang, Jianguo Cao, Zirui Guo
}

\begin{abstract}
In this paper, a new structure of a lower extremity exoskeleton robot is proposed, the knee joint of which adopts a five-link gear mechanism to complete the transmission, thus realizing a multi-center rotational motion at the knee. By establishing the kinematic model and dynamic model of this robot, this paper provides a theoretical analysis of the proposed structure and uses MATLAB to solve the motion trajectory of the ankle joint in the spatial sagittal plane to verify the correctness of the kinematic model. Based on the simulation analysis of the human-machine system model performed in ADAMS, the torque characteristics and rotational speed characteristics of each joint motor deceleration module are solved. Furthermore, the simulation results are compared with the theoretical results to verify the correctness of the dynamic model, and the theoretical basis is provided for the implementation of the concrete prototype experiment of the lower extremity exoskeleton robot. text alignment should be justified. References and Author's Profile must be in Font Size 8 Hanging 0.25 with single line spacing.
\end{abstract}

Index Terms - exoskeleton robot; kinematics; dynamics; simulation

\section{INTRODUCTION}

With an increasing aging population and the occurrence of accidents, a large number of patients with lower limb motor dysfunction present across the world every year. The lack of mobility greatly reduces the quality of life of these patients, and some patients may even experience a variety of complications as a result of sitting in a wheelchair for a long time. According to clinical hospital studies, some patients can re-stand and walk after a period of rehabilitation training, and are even expected to return to a healthy state $[1,2]$. Lower extremity exoskeleton robots are human-computer composite systems that integrate mechatronics, biology, medical rehabilitation engineering, and so on, which can be worn on a human body to assist the wearer to stand, walk, and perform other activities. At the same time, it can be used for the rehabilitation training of patients with lower limb sports injury, improving the shortcomings of traditional treatment methods and reducing the workload of treatment physicians.

Shufeng Tang, College of mechanical engineering, Inner Mongolia uni versity of technology Hohhot, China

Jianguo Cao, College of mechanical engineering, Inner Mongolia university of technology Hohhot, China.

Zirui Guo, College of mechanical engineering, Inner Mongolia university of technology Hohhot, China.
The BLEEX and HULC, developed by Berkeley Bionics, used a linear hydraulic actuator to locate the knee drive in the thigh area. This device has the advantages of a simple structure and easy installation and maintenance. However, when a linear hydraulic actuator is used for rotational motion, the movement range and torque of the joint are limited by the connection position of the linear actuator $[3,4]$. When the human body performs the actual movement, the extension/flexion movement of the knee joint is not simply single-axis rotational motion; rather, the instantaneous rotation center of its motion is accompanied by a certain position slip, namely, multi-center rotational motion [5]. Dong and others developed the exoskeleton robot knee joint using a double four-link mechanism. Because the motion through the thigh part of the four-link mechanism to drive the four-link mechanism at the knee to achieve multi-center rotation movement at the knee is more consistent with the actual movement of the human knee joint, and its structure enhances the movement stability of the knee joint [6].

This paper first proposes a new structure of a lower extremity exoskeleton robot. The knee joint uses a five-link gear mechanism to complete the transmission, which can realize multi-center rotational motion at the knee. The theoretical analysis of the kinematic model and the dynamic model are carried out, and the trajectory of the ankle joint in the sagittal plane of the ankle is solved using MATLAB to verify the correctness of the kinematic model. ADAMS is used to simulate and analyze the human-machine system model, and the torque characteristics and rotational speed characteristics of each joint motor deceleration module of the human-machine system are solved. Lastly, the simulation results are compared with the theoretical calculation results.

\section{STRUCTURAL DESIGN PRINCIPLE OF LOWER EXTREMITY EXOSKELETON ROBOT}

In this paper, the human body with a height of $170 \mathrm{~cm}$ and a weight of $70 \mathrm{~kg}$ is used as a reference object. The dimensions of the parts of the lower extremity exoskeleton robot are set as: foot-ankle length $l_{1}=66 \mathrm{~mm}$, ankle-knee length (adjustable) $l_{2}$ $=372 \mathrm{~mm}$, knee-hip length (adjustable) $l_{3}=463 \mathrm{~mm}$. Since the human-machine system travels in a straight manner on flat ground, it mainly manifests the movement of the sagittal plane of the human body. Therefore, when the structure of the lower extremity exoskeleton robot was designed, the motion of the sagittal plane was mainly considered. According to gait data $[7,8]$, obtained from the observation of a person walking along a straight line at a speed of $0.8 \mathrm{~m} / \mathrm{s}$, and the sitting posture of the human body, the degrees of freedom of the joints of the exoskeleton robot and the range of motion were set as shown in Table 1. 
Table 1. Freedom configuration and motion range of each joint of the lower extremity exoskeleton.

\begin{tabular}{|c|c|c|c|}
\hline Joint & Freedom & $\begin{array}{l}\text { Assist } \\
\text { range }\end{array}$ & $\begin{array}{c}\text { Motion } \\
\text { range }\end{array}$ \\
\hline Hip & $\begin{array}{c}\text { Extension/flexion } \\
\text { Abduction/abduction } \\
\text { External } \\
\text { rotation/internal } \\
\text { rotation }\end{array}$ & $\begin{array}{c}30^{\circ} \sim-21 \\
\circ \\
- \\
-\end{array}$ & $\begin{array}{l}100^{\circ} \sim-30 \\
\circ \\
10^{\circ} \sim-10^{\circ} \\
10^{\circ} \sim-10^{\circ}\end{array}$ \\
\hline Knee & Extension/flexion & $0^{\circ} \sim-68^{\circ}$ & $0^{\circ} \sim-90^{\circ}$ \\
\hline Ankle & Extension/flexion & - & $20^{\circ} \sim-20^{\circ}$ \\
\hline
\end{tabular}

The mechanical structure of the lower extremity exoskeleton robot designed in this paper is shown in Figure 1, which includes the backpack device, waist mechanism, hip joint mechanism, knee joint mechanism, ankle joint mechanism, foot mechanism, and leggings device, in which the hip and knee joint mechanism adopt the motor deceleration module active drive, the ankle joint mechanism and the waist mechanism play a passive adaptation role, makes the device more comfortable to wear and flexible in movement.

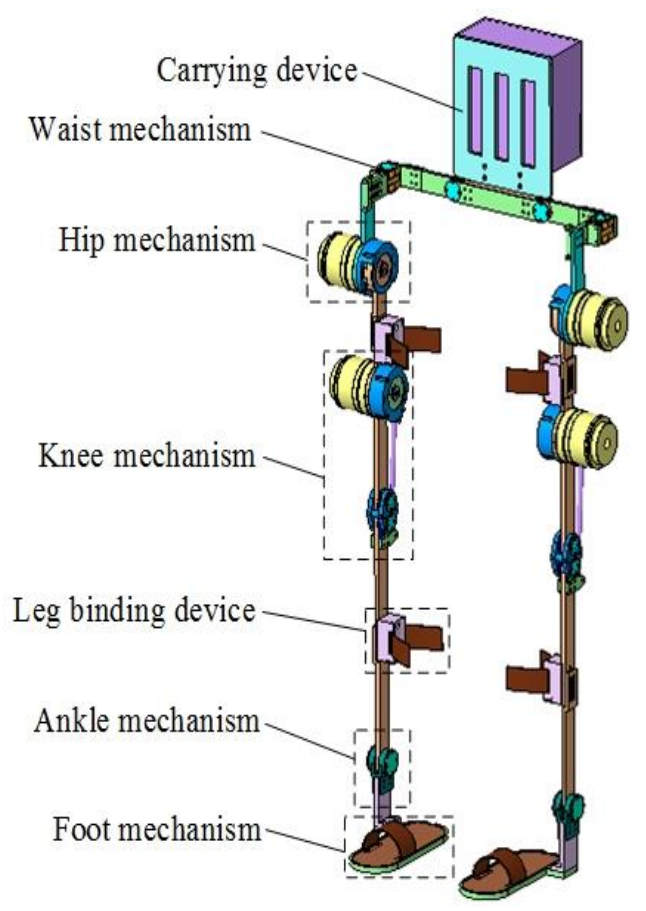

Figure 1. Mechanical structure of the lower extremity exoskeleton robot.

The specific structure of the knee joint mechanism is shown in Figure 2. Its motor deceleration module is located in the middle of the thigh to achieve stretching/buckling movement at the knee through a set of connected rod-gear transmission mechanisms. This scheme effectively reduces the quality of the following parts of the knee of the human-machine system, and can adapt to the actual movement of the robot, which is closer to the actual movement of the human knee. The purpose of the lower extremity exoskeleton robot designed in this paper is mainly to help the wearer to walk along a straight line, so the power range of the knee joint was set to $0^{\circ} \sim-68^{\circ}$. Considering the sitting position of the human-machine system, the maximum motion range of the knee joint was set to $0^{\circ} \sim-90^{\circ}$ and a hard limit protection shell was designed.

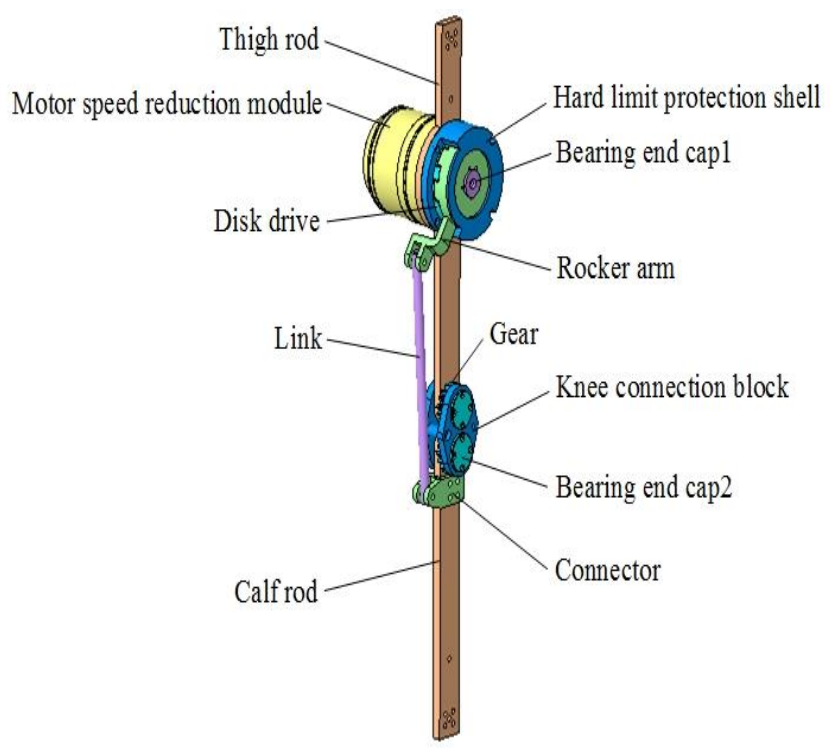

Figure 2. Knee joint mechanism.

\section{KINEMATICS AND DYNAMICS ANALYSES}

\section{A. Kinematics analysis}

First, we established the kinematic model of the human-machine system. The Denavit-Hartenberg (D-H) coordinate method was proposed by Denavit and Hartenberg to attach a reference coordinate system to each rod. This method can conveniently define the geometric relation of each rod of different kinds of robot $[9,10]$ using four parameters to determine the posture of one coordinate system relative to the other coordinate system. The four parameters are: connecting rod length $l_{i}$, connecting rod torsion angle $\alpha_{i}$, joint offset $h_{i}$, and joint corner $\theta_{i}$.

Because this paper mainly studies the motion of the robot on the sagittal plane, it could be reduced to a planar seven-link rigid body system. The $\mathrm{D}-\mathrm{H}$ coordinate method was used to establish the various coordinate systems of the human-machine system as shown in Figure 3. The specific agreement is as follows:

(1) The $N$ moving rods in the robot mechanism are numbered from 1 to $N$; the ground number is 0 ; the $N$ joints of the robot are numbered from 1 to $N$, and joint $i$ is arranged between the connecting rod $(i-1)$ and $i$, the $O_{0}$ origin of the ground coordinate system is established at the ankle joint of the supporting leg, which coincides with the origin of the coordinate system $O_{1}$; the $z_{i}$ axis is arranged on the axis of joint $i$, and the $x_{i-1}$ axis is arranged on the cylindrical of the $z_{i-1}$ axis and the $z_{i}$ axis.

(2) The length of the rod is $l_{i}$, which is the distance from the $z_{i-1}$ shaft to the $z_{i}$ shaft along the $x_{i-1}$ axis; the torsion angle of the connecting rod $\alpha_{i}$ is the angle of rotation from the $x_{i-1}$ axis to the $z_{i-1}$ axis around the $z_{i}$ axis; the joint offset $h_{i}$ is the distance from the $z_{i}$ axis to the $x_{i}$ axis along the $x_{i-1}$ axis; the joint corner $\theta_{i}$ the angle of rotation along the $z_{i}$ axis from the $x_{i-1}$ axis to the $x_{i}$ axis. 


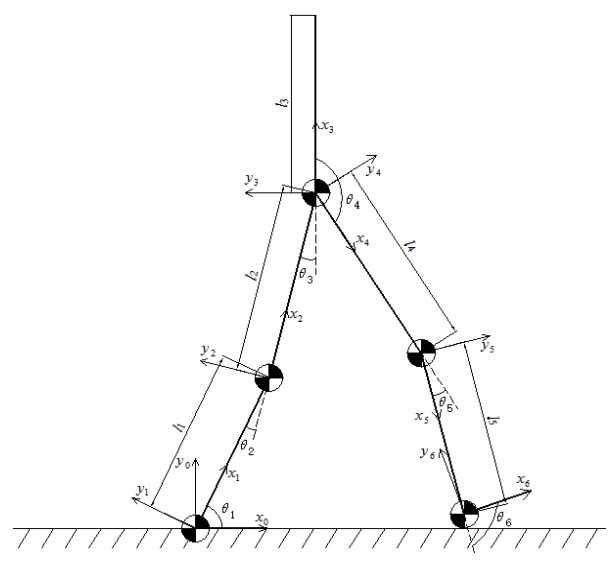

Figure 3. Denavit-Hartenberg $(\mathrm{D}-\mathrm{H})$ coordinate model of the lower extremity exoskeleton robot.

From the above agreement, the positioning of the reference coordinate system $i$ relative to the reference coordinate system $(i-1)$ can be realized, and Equation (1) is used to represent the transformation matrix between the rods:

$$
{ }^{i-1} T_{i}=\operatorname{Rot}\left(x_{i-1}, \alpha_{i-1}\right) \operatorname{Trans}\left(x_{i-1}, l_{i-1}\right) \operatorname{Rot}\left(z_{i}, \theta_{i}\right) \operatorname{Trans}\left(z_{i}, h_{i}\right)
$$

The equivalent homogeneous transformation calculated can be obtained as follows:

$$
{ }^{i-1} T_{i}=\left[\begin{array}{cccc}
c_{\theta i} & -s_{\theta i} & 0 & l_{i-1} \\
s_{\theta i} c_{\alpha i-1} & c_{\theta i} c_{\alpha i-1} & -s_{\alpha i-1} & -s_{\alpha i-1} h_{i} \\
s_{\theta i} s_{\alpha i-1} & c_{\theta i} s_{\alpha i-1} & c_{\alpha i-1} & c_{\alpha i-1} h_{i} \\
0 & 0 & 0 & 1
\end{array}\right]
$$

where:

$$
\begin{gathered}
c_{\theta i}=\cos \theta_{i}, \quad c_{\alpha i-1}=\cos \alpha_{i-1} ; \\
s_{\theta i}=\sin \theta_{i}, \quad s_{\alpha i-1}=\sin \alpha_{i-1} .
\end{gathered}
$$

The homogeneous coordinate transformation matrix between the various rods of the robot are listed as follows:

$$
\begin{aligned}
{ }^{0} T_{1} & =\left[\begin{array}{cccc}
c_{\theta 1} & -s_{\theta 1} & 0 & 0 \\
s_{\theta 1} & c_{\theta 1} & 0 & 0 \\
0 & 0 & 1 & 0 \\
0 & 0 & 0 & 1
\end{array}\right] \\
{ }^{1} T_{2} & =\left[\begin{array}{cccc}
c_{\theta 2} & -s_{\theta 2} & 0 & l_{1} \\
s_{\theta 2} & c_{\theta 2} & 0 & 0 \\
0 & 0 & 1 & 0 \\
0 & 0 & 0 & 1
\end{array}\right] \\
{ }^{2} T_{3} & =\left[\begin{array}{cccc}
c_{\theta 3} & -s_{\theta 3} & 0 & l_{2} \\
s_{\theta 3} & c_{\theta 3} & 0 & 0 \\
0 & 0 & 1 & 0 \\
0 & 0 & 0 & 1
\end{array}\right] \\
{ }^{3} T_{4} & =\left[\begin{array}{cccc}
c_{\theta 4} & -s_{\theta 4} & 0 & 0 \\
s_{\theta 4} & c_{\theta 4} & 0 & 0 \\
0 & 0 & 1 & 0 \\
0 & 0 & 0 & 1
\end{array}\right]
\end{aligned}
$$

$$
\begin{aligned}
{ }^{4} T_{5}= & {\left[\begin{array}{cccc}
c_{\theta 5} & -s_{\theta 5} & 0 & l_{4} \\
s_{\theta 5} & c_{\theta 5} & 0 & 0 \\
0 & 0 & 1 & 0 \\
0 & 0 & 0 & 1
\end{array}\right] } \\
{ }^{5} T_{6}= & {\left[\begin{array}{cccc}
c_{\theta 6} & -s_{\theta 6} & 0 & l_{5} \\
s_{\theta 6} & c_{\theta 6} & 0 & 0 \\
0 & 0 & 1 & 0 \\
0 & 0 & 0 & 1
\end{array}\right] }
\end{aligned}
$$

Then the transformation matrix of the ankle joint coordinate system of the swinging leg relative to the ground coordinate system is obtained:

$$
\begin{aligned}
{ }^{0} T_{6} & ={ }^{0} T_{1}{ }^{1} T_{2}{ }^{2} T_{3}{ }^{3} T_{4}{ }^{4} T_{5}{ }^{5} T_{6} \\
& =\left[\begin{array}{cccc}
c_{\theta 123456} & -s_{\theta 123456} & 0 & l_{1} c_{\theta 1}+l_{2} c_{\theta 12}+l_{4} c_{\theta 1234}+l_{5} c_{\theta 12345} \\
s_{\theta 123456} & c_{\theta 123456} & 0 & l_{1} s_{\theta 1}+l_{2} s_{\theta 12}+l_{4} s_{\theta 1234}+l_{5} s_{\theta 12345} \\
0 & 0 & 1 & 0 \\
0 & 0 & 0 & 1
\end{array}\right]
\end{aligned}
$$

The coordinates of the ankle joint on the sagittal plane of the swinging leg are as follows:

$$
\left\{\begin{array}{l}
x_{6}=l_{1} c_{\theta 1}+l_{2} c_{\theta 12}+l_{4} c_{\theta 1234}+l_{5} c_{\theta 12345} \\
y_{6}=l_{1} s_{\theta 1}+l_{2} s_{\theta 12}+l_{4} s_{\theta 1234}+l_{5} s_{\theta 12345}
\end{array}\right.
$$

where:

$$
\begin{aligned}
& c_{\theta 12 \ldots \mathrm{n}}=\cos \left(\theta_{1}+\theta_{2}+\ldots+\theta_{n}\right) ; \\
& s_{\theta 12 \ldots \mathrm{n}}=\sin \left(\theta_{1}+\theta_{2}+\ldots+\theta_{n}\right) .
\end{aligned}
$$

By inserting the angle value of the human body into Equation (10), the correctness of the kinematic model of the lower extremity exoskeleton human-machine system established in this paper can be verified. The motion range of each joint set in Table 1 is put into Equation (10), and MATLAB is used to obtain the motion range of the swinging leg ankle joint in the spatial sagittal plane as shown in Figure 4. As seen in the figure, the motion range of the ankle joint in space is basically consistent with the actual situation, which fully proves the correctness of the kinematic model of the human-machine system established in this paper.

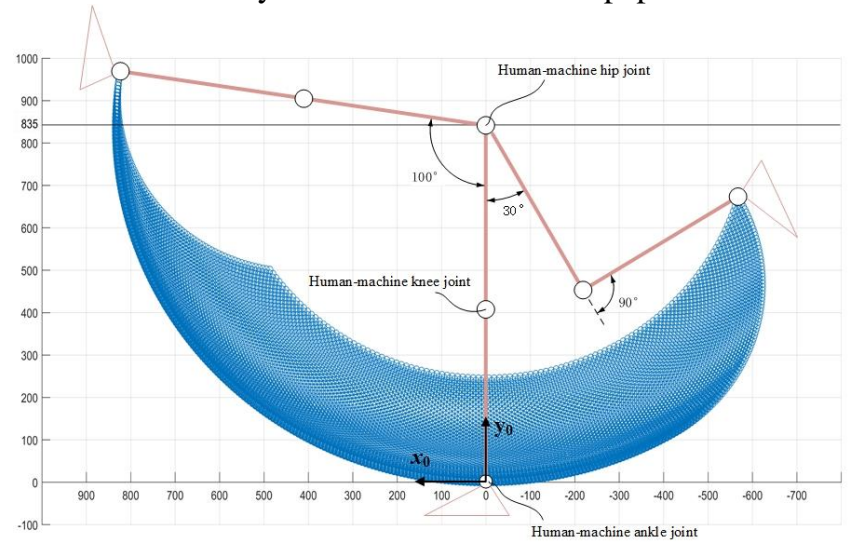

Figure 4. Motion range of the swinging leg ankle joint in the spatial sagittal plane. 
In order to reflect the kinematics characteristics of the system more accurately, it was also necessary to establish the kinematic relationship between the knee joint mechanism and the human-machine system. As shown in Figure 5, the kinematic model was established on the basis of the motion diagram of the knee joint mechanism.

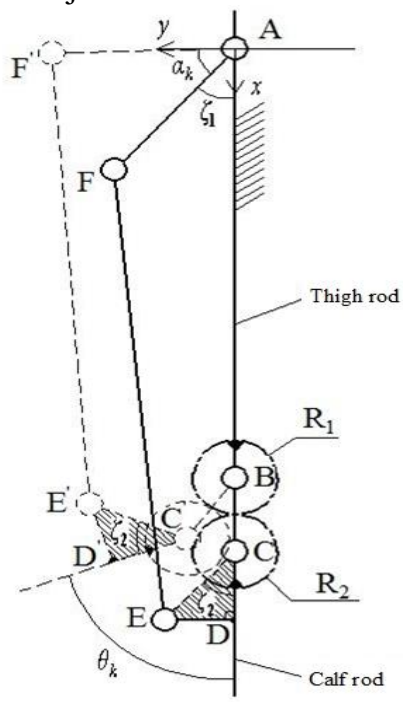

Figure 5. Kinematic model of the knee joint mechanism.

As shown in Figure 5, $\zeta_{1}$ represents the initial mounting position angle of rod AF; $\zeta_{2}$ is the angle between rod $\mathrm{CD}$ and auxiliary line $\mathrm{CE}$; rod $\mathrm{AF}$ is fixed with the output end of the motor deceleration module; $\alpha_{k}$ indicates the angle at which $\operatorname{rod} \mathrm{AF}$ turns relative to its initial position during motion; and $\theta_{k}$ indicates the angle at which the calf rod turns relative to the thigh rod. The relationships among these variables are as follows:

$$
\tan \zeta_{2}=\frac{l_{D E}}{l_{C D}}
$$

So,

$$
\zeta_{2}=\arctan \frac{l_{D E}}{l_{C D}}
$$

Gears $R_{1}$ and $R_{2}$ are respectively fixed to the knee joint mechanism of the thigh rod and the calf rod, and $R_{1}=R_{2}$. In motion, $\mathrm{R}_{2}$ rotates around $\mathrm{R}_{1}$, and it also rotates around axis C. The relationship is expressed as follows:

$$
\angle \mathrm{CBC}^{\prime}=\frac{\theta_{k}}{2}
$$

A planar rectangular coordinate system was established with $\mathrm{A}$ as the original point, as shown in Figure 5, and the hinge contact coordinates in the figure are:

$$
\begin{aligned}
& \left\{\begin{array}{l}
x_{A}=0 \\
y_{A}=0
\end{array}\right. \\
& \left\{\begin{array}{l}
x_{B}=l_{A B} \\
y_{B}=0
\end{array}\right. \\
& \left\{\begin{array}{l}
x_{C}=l_{B C} \cos \frac{\theta_{k}}{2}+l_{A B} \\
y_{C}=l_{B C} \sin \frac{\theta_{k}}{2}
\end{array}\right.
\end{aligned}
$$

$$
\begin{aligned}
& \left\{\begin{array}{l}
x_{E}=l_{C E} \cos \left(\theta_{k}+\zeta_{2}\right)+l_{B C} \cos \frac{\theta_{k}}{2}+l_{A B} \\
y_{E}=l_{C E} \sin \left(\theta_{k}+\zeta_{2}\right)+l_{B C} \sin \frac{\theta_{k}}{2}
\end{array}\right. \\
& \left\{\begin{array}{l}
x_{F}=l_{A F} \cos \left(\alpha_{k}+\zeta_{1}\right) \\
y_{F}=l_{A F} \sin \left(\alpha_{k}+\zeta_{1}\right)
\end{array}\right.
\end{aligned}
$$

due to the relationship expressed below:

$$
l_{E F}=\sqrt{\left(x_{E}-x_{F}\right)^{2}+\left(y_{E}-y_{F}\right)^{2}}
$$

By substituting the coordinates of Equation (14) into Equation (12), we obtain:

$$
\begin{aligned}
l_{E F}^{2}= & {\left[l_{C E} \cos \left(\theta_{k}+\arctan \frac{l_{D E}}{l_{C D}}\right)+l_{B C} \cos \frac{\theta_{k}}{2}+l_{A B}-l_{A F} \cos \left(\alpha_{k}+\varnothing_{1}\right)\right]^{2} } \\
& +\left[l_{C E} \sin \left(\theta_{k}+\arctan \frac{l_{D E}}{l_{C D}}\right)+l_{B C} \sin \frac{\theta_{k}}{2}-l_{A F} \sin \left(\alpha_{k}+\varnothing_{1}\right)\right]^{2}
\end{aligned}
$$

From the above analysis, it is assumed that the initial human-machine system is in an upright state. This is to say, the calf is collinear with the thigh (i.e., $\theta_{k}=0^{\circ}$ ). Then, the size of each member of the knee joint mechanism is inserted into Equation (15), and the walking assistance process can be obtained by calculation and rounding. The angle of rod AF varies from $-38^{\circ}$ to $-83^{\circ}$, and the angle of the output of the motor reduction module ranges from $0^{\circ}$ to $-45^{\circ}$.

\section{B. Dynamics Theory analysis}

At present, dynamic analysis methods of multi-rigid systems are more commonly used, such as the Lagrange equation method and the Newton-Euler method. The Lagrange method has the advantages of not requiring internal force to be solved, simple derivation, and a compact structure, so the dynamics analysis of external skeletal robots is often preferred. The Lagrange method is based on the differential of the energy item to the system variables and time, which takes the human-machine system as a whole and uses independent generalized coordinates to complete the establishment of the system dynamics equation [11].

This article first defines the Lagrange functions of a multi-rigid system as follows:

$$
L=E_{\mathrm{T}}-E_{\mathrm{U}}
$$

where:

$E_{\mathrm{T}}-$ the total kinetic energy of the system;

$E_{\mathrm{U}}$ - the total potential energy of the system.

Further, using the Lagrange equation for each generalized coordinate, the general expansion formula of the kinetic equation can be expressed as follows:

$$
\tau_{i}=\frac{d}{d t} \frac{\partial L}{\partial \dot{q}_{i}}-\frac{\partial L}{\partial q_{i}}
$$

where:

$\tau_{i}$ - the generalized external force vector (including force or torque, which depends on the nature of $q_{i}$; when $q_{i}$ is the amount of rotation, $\tau_{i}$ represents the torque, and when $q_{i}$ is elongated, $\tau_{i}$ represents the force);

$q_{i}$ - the generalized coordinate vectors. 
In this paper, the dynamic model of the human-machine system was first established when the dynamics analysis of the knee joint mechanism was carried out. Because this paper mainly focuses on motion on the sagittal plane, the human-machine system could be reduced to a planar seven-link rigid system. When the human body walks in a straight line along a flat surface, the two modes of single-legged support state and two-legged support state are included in the gait period according to the contact between the soles of the feet and the ground. So, two kinetic models were established in this paper, and the dynamics of the two different states were analyzed [12].

a) Dynamic modeling of single-legged support state

As shown in Figure 6, the dynamic model of the human-machine system in the single-legged support state assumes that the hind leg is supported while the front leg swings.

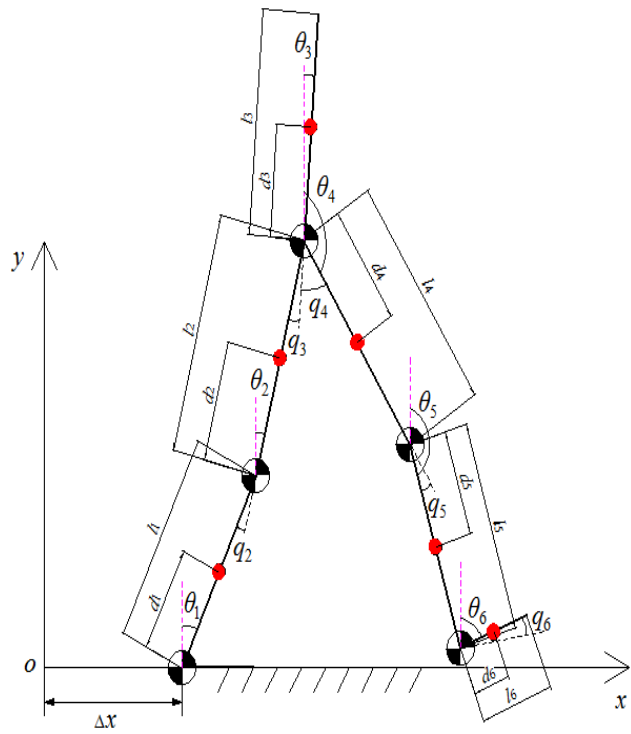

Figure 6. Dynamic model of the single-legged support state in the human-machine system.

In Figure $6, l_{1}-l_{6}$ respectively represent the length of the calf, the length of the posterior thigh, the length of the torso, the length of the anterior thigh, the length of the anterior calf, the length of the right foot, and the distance between the joints and the corresponding centroid. $\theta_{i}$ is the angle between the rods and the $y$ axis, and $q_{i}$ is the angle between the adjacent rods. The relationships among these variables are as follows:

$$
\begin{aligned}
& q_{1}=\theta_{1} ; \\
& q_{2}=\theta_{2}-\theta_{1} ; \\
& q_{3}=\theta_{3}-\theta_{2} ; \\
& q_{4}=\theta_{3}-\theta_{4}+\pi ; \\
& q_{5}=\theta_{4}-\theta_{5} ; \\
& q_{6}=\theta_{6}-\theta_{5}+\frac{\pi}{2} .
\end{aligned}
$$

The centroid coordinates of each rod in the figure can be expressed as:

$$
\left\{\begin{array}{l}
x_{i}=\Delta x+d_{i} \sin \theta_{i}+\sum_{j=1}^{i-1}\left(a_{j} l_{j} \sin \theta_{j}\right) \\
y_{i}=d_{i} \cos \theta_{i}+\sum_{j=1}^{i-1}\left(a_{j} l_{j} \cos \theta_{j}\right)
\end{array}\right.
$$

where:

$$
a_{j}= \begin{cases}0 & j=3 \\ 1 & j=1,2,4,5,6\end{cases}
$$

The centroid velocity can be expressed as:

$$
\left\{\begin{array}{l}
\dot{x}_{i}=d_{i} \dot{\theta}_{i} \cos \theta_{i}+\sum_{j=1}^{i-1}\left(a_{j} l_{j} \dot{\theta}_{j} \cos \theta_{j}\right) \\
\dot{y}_{i}=-d_{i} \dot{\theta}_{i} \sin \theta_{i}-\sum_{j=1}^{i-1}\left(a_{j} l_{j} \dot{\theta}_{j} \sin \theta_{j}\right)
\end{array} .\right.
$$

The total kinetic energy of the system can be expressed as:

$$
\begin{aligned}
E_{T} & =\sum_{i=1}^{6} \frac{1}{2}\left[m_{i}\left(\dot{x}_{i}^{2}+\dot{y}_{i}^{2}\right)+I_{i} \dot{\theta}_{i}^{2}\right] \\
& =\sum_{i=1}^{6} \frac{1}{2}\left[\left(I_{i}+m_{i} d_{i}^{2}\right) \dot{\theta}_{i}^{2}\right]+\sum_{i=1}^{6}\left\{m_{i} d_{i} \dot{\theta}_{i}\left\{\sum_{j=1}^{i-1}\left[a_{j} l_{j} \dot{\theta}_{j} \cos \left(\theta_{i}-\theta_{j}\right)\right]\right\}\right\} \\
& +\sum_{i=1}^{6}\left\{\frac{1}{2} m_{i}\left\{\left[\sum_{j=1}^{i-1}\left(a_{j} l_{j} \dot{\theta}_{j} \cos \theta_{j}\right)\right]^{2}+\left[\sum_{j=1}^{i-1}\left(a_{j} l_{j} \dot{\theta}_{j} \sin \theta_{j}\right)\right]^{2}\right]\right\}
\end{aligned}
$$

The total potential energy of the system can be expressed as:

$$
E_{U}=\sum_{i=1}^{6} m_{i} g y_{i}=\sum_{i=1}^{6}\left\{m_{i} g\left[\sum_{j=1}^{i-1}\left(a_{j} l_{j} \cos \theta_{j}\right)+d_{i} \cos \theta_{i}\right]\right\}
$$

The kinetic equation for the support state of a single leg according to the Lagrange equation is as follows:

$$
\mathrm{T}=\mathrm{H}(\theta) \ddot{\theta}+C(\theta, \dot{\theta}) \dot{\theta}+\tau_{g}(\theta)
$$

where $\mathrm{H}$ is a $6 \times 6$ generalized inertial matrix; $C$ is a $6 \times 6$ centrifugal force matrix; and $\tau_{g}$ is a $6 \times 1$ gravity matrix. The specific forms of these are as follows:

$$
\left\{\begin{array}{l}
H_{i j}=p_{i j} \cos \left(\theta_{i}-\theta_{j}\right) \\
C_{i j}=p_{i j} \sin \left(\theta_{i}-\theta_{j}\right) \dot{\theta}_{j} \\
\tau_{g i}=g_{i} \sin \theta_{i}
\end{array}\right.
$$

where $i, j=1,2,3,4,5,6$;

$$
\begin{aligned}
& p_{i j}=\left\{\begin{array}{cc}
I_{i}+m_{i} d_{i}^{2}+a_{i}\left(\sum_{j=i+1}^{6} m_{j}\right) l_{i}^{2} & j=i \\
a_{i} m_{j} d_{j} l_{i}+a_{i} a_{j}\left(\sum_{k=j+1}^{6} m_{k}\right) l_{i} l_{j} & j>i ; \\
P_{j i} & j<i
\end{array}\right. \\
& g_{i}=m_{i} d_{i} g+a_{i}\left(\sum_{j=i+1}^{6} m_{j}\right) l_{i} g \text {. }
\end{aligned}
$$


As mentioned above, the relationship between qi and $\theta_{i}$ can be expressed as:

$$
q=\mathrm{X} \theta+\mathrm{Y}
$$

where:

$$
\mathrm{X}=\left[\begin{array}{cccccc}
1 & 0 & 0 & 0 & 0 & 0 \\
-1 & 1 & 0 & 0 & 0 & 0 \\
0 & -1 & 1 & 0 & 0 & 0 \\
0 & 0 & 1 & -1 & 0 & 0 \\
0 & 0 & 0 & 1 & -1 & 0 \\
0 & 0 & 0 & 0 & -1 & 1
\end{array}\right] ; \quad \mathrm{Y}=\left[\begin{array}{c}
0 \\
0 \\
0 \\
\pi \\
0 \\
\frac{\pi}{2}
\end{array}\right]
$$

There is a relationship below:

$$
\theta=\mathrm{X}^{-1}(\boldsymbol{q}-\mathrm{Y})
$$

The expression of each joint moment is:

$$
\tau_{i}=\sum_{j=1}^{6} \mathrm{~T}_{j} \frac{\partial \theta_{j}}{\partial q_{i}}=\sum_{j=1}^{6} \mathrm{~T}_{j}\left(\mathrm{X}^{-1}\right)_{j i}
$$

b) Dynamic modeling of two-legged support state

As shown in Figure 7, the dynamic model of the human-machine system in the two-legged support state is characterized by both legs being in contact with the ground to support the whole system together.

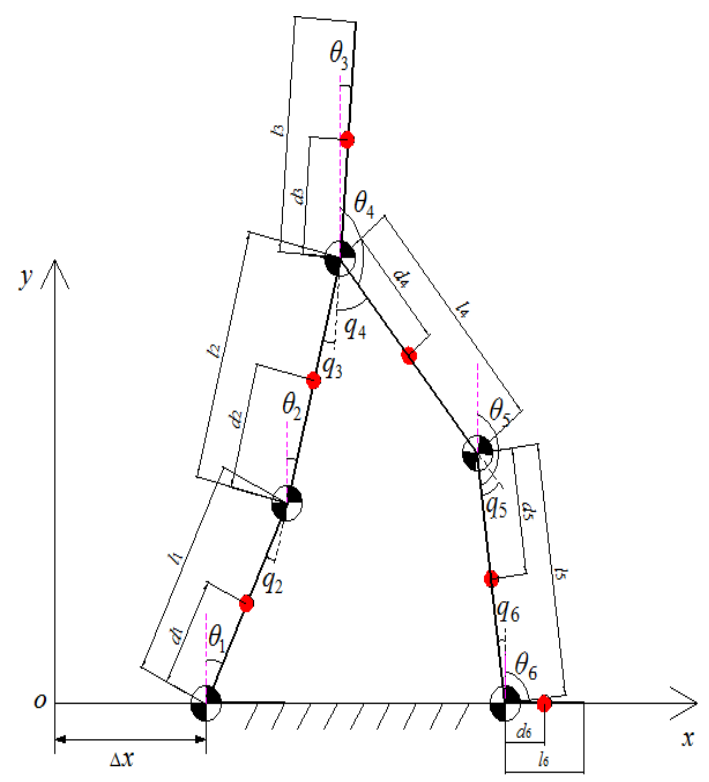

Figure 7. Dynamic model of the two-legged support state in the human-machine system.

Because the movement of the upper body of the system is slow and almost constant, it can be regarded as static in uniform linear motion. In the analysis, the mass of the upper body is divided into two parts and the whole kinetic model is decomposed into two single-legged rigid connecting rod systems, that is, the hind leg connecting rod system and the front leg connecting rod system, as shown in Figure 8.
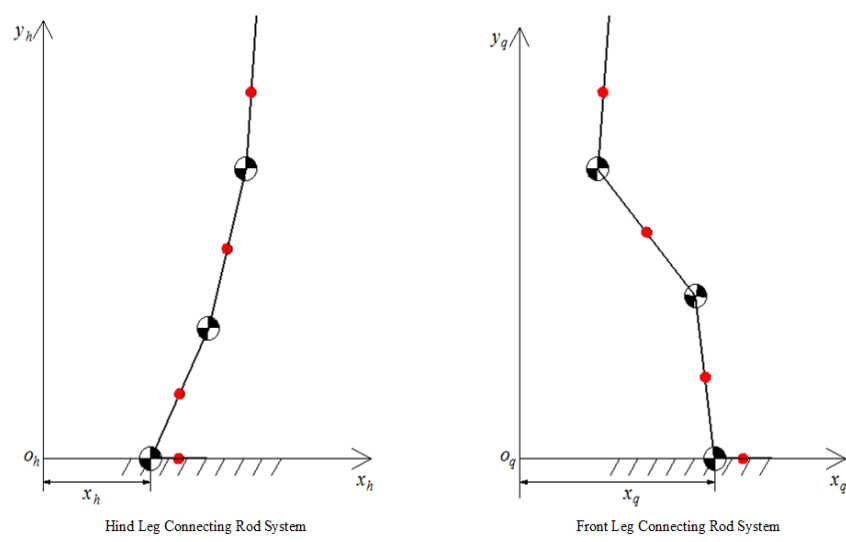

Figure 8. Two single-legged rigid connecting rod systems.

The mass of the upper body acting on the foreleg is defined as $m_{3 q}$, and the mass of the hind leg as $m_{3 h}$; their respective sizes are related to the distance between the forefoot and the $l_{3}$ centroid of the rod on the $x$ axis $x_{3 q}$ and the distance between the rear foot and the $l_{3}$ centroid of the rod on the $x$ axis. Thus, we obtain the following relationships:

$$
\begin{gathered}
\left\{\begin{array}{l}
m_{3}=m_{3 q}+m_{3 h} \\
m_{3 q} \cdot x_{3 q}=m_{3 h} \cdot x_{3 h}
\end{array}\right. \\
\left\{\begin{array}{l}
x_{3 h}=x_{h}+l_{1} \sin \theta_{1}+l_{2} \sin \theta_{2}+d_{3} \sin \theta_{3} \\
x_{3 q}=x_{q}-l_{5} \sin \theta_{5}-l_{4} \sin \theta_{4}+d_{3} \sin \theta_{3}
\end{array}\right.
\end{gathered}
$$

For the hind leg connecting rod system, there are the following relationships:

$$
\begin{aligned}
& q_{1}=\theta_{1} \\
& q_{2}=\theta_{2}-\theta_{1} \\
& q_{3}=\theta_{3}-\theta_{2} .
\end{aligned}
$$

The centroid coordinates of its rods can be expressed as:

$$
\left\{\begin{array}{l}
x_{i}=\Delta x+d_{i} \sin \theta_{i}+\sum_{j=1}^{i-1}\left(l_{j} \sin \theta_{j}\right) \\
y_{i}=d_{i} \cos \theta_{i}+\sum_{j=1}^{i-1}\left(l_{j} \cos \theta_{j}\right)
\end{array}\right.
$$

The centroid velocity of each rod can be expressed as:

$$
\left\{\begin{array}{l}
\dot{x}_{i}=d_{i} \dot{\theta}_{i} \cos \theta_{i}+\sum_{j=1}^{i-1}\left(l_{j} \dot{\theta}_{j} \cos \theta_{j}\right) \\
\dot{y}_{i}=-d_{i} \dot{\theta}_{i} \sin \theta_{i}-\sum_{j=1}^{i-1}\left(l_{j} \dot{\theta}_{j} \sin \theta_{j}\right)
\end{array}\right.
$$

The total kinetic energy of the hind leg connecting rod system is obtained as:

$$
\begin{aligned}
E_{\mathrm{T} h} & =\sum_{i=1}^{3} \frac{1}{2}\left[m_{i}\left(\dot{x}_{i}^{2}+\dot{y}_{i}^{2}\right)+I_{i} \dot{\theta}_{i}^{2}\right] \\
& =\sum_{i=1}^{3} \frac{1}{2}\left[\left(I_{i}+m_{i} d_{i}^{2}\right) \dot{\theta}_{i}^{2}\right]+\sum_{i=1}^{3}\left\{m_{i} d_{i} \dot{\theta}_{i}\left\{\sum_{j=1}^{i-1}\left[l_{j} \dot{\theta}_{j} \cos \left(\theta_{i}-\theta_{j}\right)\right]\right\}\right\} \\
& +\sum_{i=1}^{3}\left\{\frac{1}{2} m_{i}\left\{\left[\sum_{j=1}^{i-1}\left(l_{j} \dot{\theta}_{j} \cos \theta_{j}\right)\right]^{2}+\left[\sum_{j=1}^{i-1}\left(l_{j} \dot{\theta}_{j} \sin \theta_{j}\right)\right]^{2}\right]\right\}
\end{aligned}
$$

The total potential energy of the hind leg connecting rod system is: 
$E_{\mathrm{U} h}=\sum_{i=1}^{3} m_{i} g y_{i}=\sum_{i=1}^{3}\left\{m_{i} g\left[\sum_{j=1}^{i-1}\left(l_{j} \cos \theta_{j}\right)+d_{i} \cos \theta_{i}\right]\right\}$

The kinetic equation of the hind leg connecting rod system obtained by the Lagrange equation is:

$$
\mathrm{T}_{h}=\mathrm{H}_{h}(\theta) \ddot{\theta}+C_{h}(\theta, \dot{\theta}) \dot{\theta}+\tau_{g h}(\theta)
$$

In the above equation, $\mathrm{H}_{h}$ is a $3 \times 3$ generalized inertial matrix; $C_{h}$ is a $3 \times 3$ centrifugal force matrix; and $\tau_{g h}$ is a $3 \times 1$ gravity matrix. The specific forms of these are as follows:

$$
\left\{\begin{array}{l}
H_{i j h}=p_{i j h} \cos \left(\theta_{i}-\theta_{j}\right) \\
C_{i j h}=p_{i j h} \sin \left(\theta_{i}-\theta_{j}\right) \theta_{j} \\
\tau_{g i h}=g_{i h} \sin \theta_{i}
\end{array}\right.
$$

where $i, j=1,2,3$;

$$
p_{i j h}=\left\{\begin{array}{cc}
I_{i}+m_{i} d_{i}^{2}+\left(\sum_{j=i+1}^{3} m_{j}\right) l_{i}^{2} & j=i \\
m_{j} d_{j} l_{i}+\left(\sum_{k=j+1}^{3} m_{k}\right) l_{i} l_{j} & j>i ; \\
P_{j i h} & j<i
\end{array}\right.
$$

The relationship between $q_{i}$ and $\theta_{i}$ is:

$$
\boldsymbol{q}=\mathrm{X}_{h} \boldsymbol{\theta}
$$

where:

$$
X_{h}=\left[\begin{array}{ccc}
1 & 0 & 0 \\
-1 & 1 & 0 \\
0 & -1 & 1
\end{array}\right]
$$

So, there is:

$$
\theta=\mathrm{X}_{h}^{-1} \boldsymbol{q}
$$

The expression of each joint movement of the hind leg connecting rod system is:

$$
\tau_{i}=\sum_{j=1}^{3} \mathrm{~T}_{j} \frac{\partial \theta_{j}}{\partial q_{i}}=\sum_{j=1}^{3} \mathrm{~T}_{j}\left(\mathrm{X}_{h}^{-1}\right)_{j i}
$$

Since the dynamic analysis process of the front leg connecting rod system is similar to that of the hind leg, there is no need to repeat it.

c) Dynamic relationship between knee joint mechanism and human-machine system

On the basis of the motion diagram of the knee joint mechanism, a dynamic model similar to its kinematic model was established, as shown in Figure 9. The line segments $a, b$, $c, d, e$ are auxiliary lines; $\gamma_{1}, \gamma_{2}, \gamma_{3}, \gamma_{4}, \beta_{1}, \beta_{2}, \beta_{3}, \beta_{4}$, as the auxiliary angles, are used in the derivation process; $\mathrm{F}_{1}$ is the output driving force of the motor deceleration module; and $\mathrm{F}_{2}$ is the transmission power of rod EF [13].

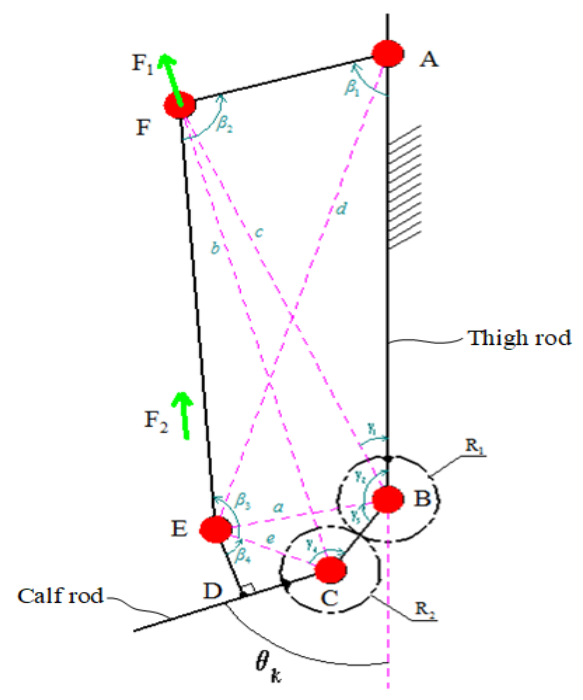

Figure 9. Kinetic model of the knee joint mechanism

Combined with the aforementioned kinematics analysis process, the following geometric relationships can be listed:

$$
\begin{aligned}
& \gamma_{1}=\cos ^{-1}\left(\frac{c^{2}+l_{A B}{ }^{2}-l_{A F}^{2}}{2 c l_{A B}}\right) \\
& \gamma_{2}=\pi-\frac{\theta_{k}}{2}-\gamma_{3} \\
& \gamma_{3}=\sin ^{-1}\left(\frac{l_{C E} \sin \gamma_{4}}{a}\right) \\
& \gamma_{4}=\pi-\tan ^{-1}\left(\frac{l_{D E}}{l_{C D}}\right)-\frac{\theta_{k}}{2} \\
& \beta_{1}=\alpha_{k}+\zeta_{1} \\
& \beta_{2}=\cos ^{-1}\left(\frac{l_{A F}^{2}+l_{E F}^{2}-d^{2}}{2 l_{A F} l_{E F}}\right) \\
& \beta_{3}=\cos ^{-1}\left(\frac{l_{E F}^{2}+e^{2}-b^{2}}{2 e l_{E F}}\right) \\
& \beta_{4}=\tan ^{-1}\left(\frac{l_{C D}}{l_{D E}}\right) \\
& a=\sqrt{l_{B C}^{2}+e^{2}-2 e l_{B C} \cos \gamma_{4}} \\
& b=\sqrt{c^{2}+l_{B C}{ }^{2}-2 c l_{B C} \cos \left(\pi-\gamma_{1}-\frac{\theta_{k}}{2}\right)} \\
& c=\sqrt{l_{A F}^{2}+l_{A B}^{2}-2 l_{A F} l_{A B} \cos \beta_{1}} \\
& d=\sqrt{a^{2}+l_{A B}{ }^{2}-2 a l_{A B} \cos \gamma_{2}} \\
& e=\sqrt{l_{C D}^{2}+l_{D E}^{2}} \text {. }
\end{aligned}
$$

The knee torque can be expressed as:

$$
\tau_{k}=\mathrm{F}_{2} l_{C D} \cos \left(\pi-\beta_{3}-\beta_{4}\right)
$$

where:

$$
\mathrm{F}_{2}=\mathrm{F}_{1} \cos \left(\beta_{2}-\frac{\pi}{2}\right)
$$

The output torque of the motor deceleration module can be expressed as:

$$
\tau_{a k}=\mathrm{F}_{1} l_{A F}
$$

So, 


$$
\tau_{a k}=\frac{l_{A F} \tau_{k}}{l_{C D} \cos \left(\beta_{2}-\frac{\pi}{2}\right) \cos \left(\pi-\beta_{3}-\beta_{4}\right)}
$$

\section{Kinematics and Dynamics Simulations}

The three-dimensional structure model of the human-machine system of the lower extremity exoskeleton was introduced into ADAMS, where the model structure was simplified. The material of each component was set to aluminum alloy, with a density of $2.82 \times 103 \mathrm{~kg} / \mathrm{m}^{3}$; the Young's modulus was $7.2 \times 104 \mathrm{MPa}$; and the Poisson's ratio was 0.33 . Then, according to the motion principle of the model structure, the corresponding motion pairs and drivers were added. In this paper, the output angle spline data of the lower extremity hip angle spline data and the first kinematics analysis obtained by MATLAB were imported into ADAMS as the driving functions of the hip and knee joint of the exoskeleton robot when it walks in a straight line on a flat surface at a speed of $0.8 \mathrm{~m} / \mathrm{s}$. The kinematics and dynamics simulations of the human-machine system model were carried out, and the simulation motion process is shown in Figure 10.

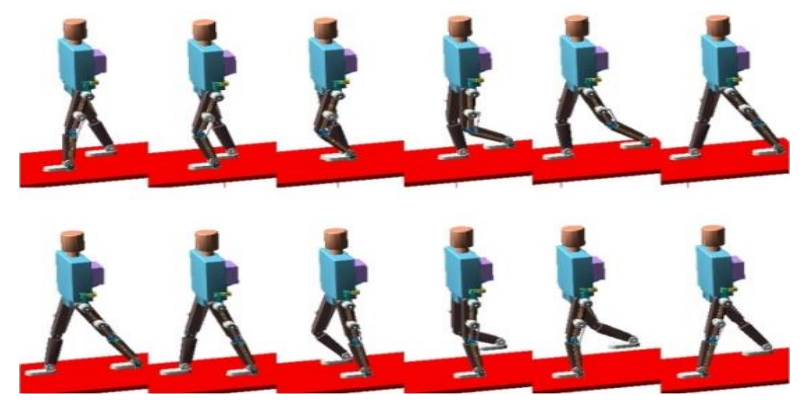

Figure 10. Simulation motion process of the human-machine system based on ADAMS.

After simulation and filtering processing, the torque-time relation curves of each joint of the lower extremity robot were obtained, and these results were compared with the theoretical calculation results through MATLAB, as shown in Figures 11 and 12.

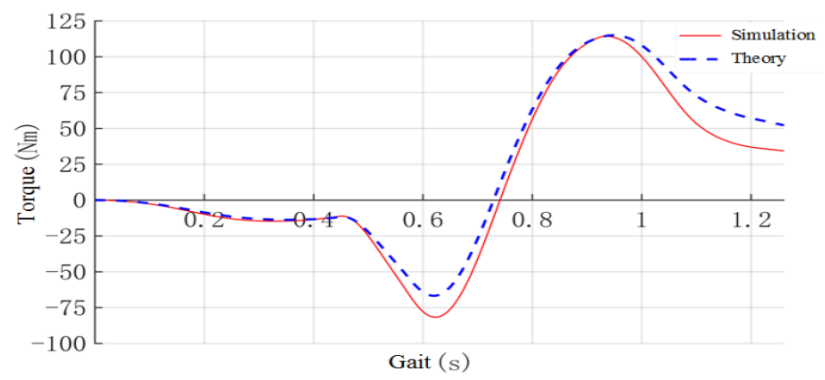

(a) Left-Hip

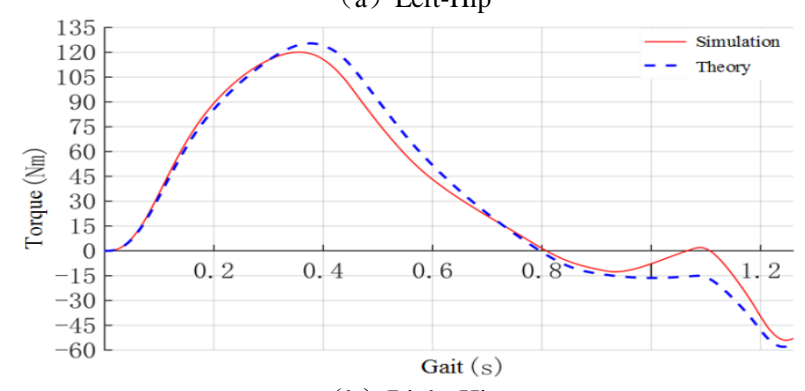

(b) Right-Hip

Figure 11. Hip torque-time relation curve.

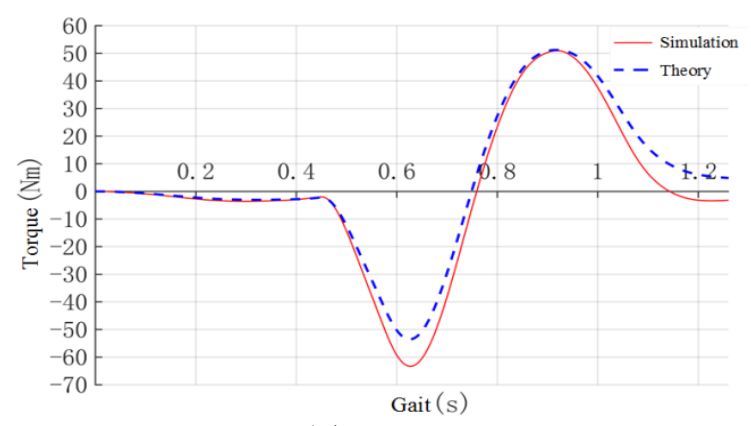

(a) Left-Knee

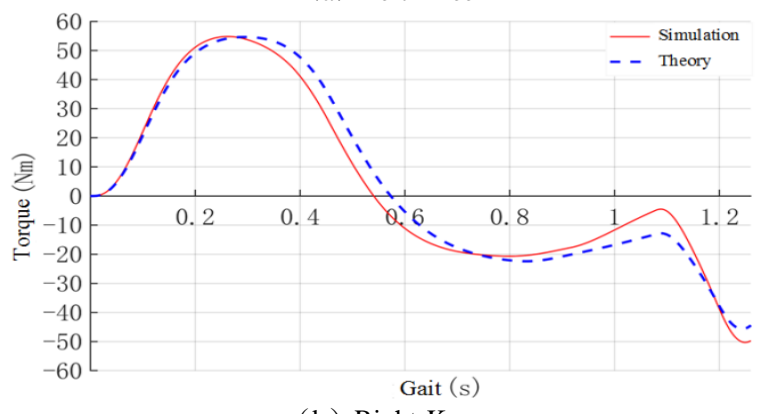

(b) Right-Knee

Figure 12. Knee torque-time relation curve.

As can be seen from Figures 11 and 12, the simulation results of the torque-time curve of each joint were somewhat biased in comparison to the theoretical calculation results, but the deviation range was small and can be regarded as reasonable deviation, so the correctness of the aforementioned dynamic analysis model was proved. The analysis of the causes of the deviation may possibly be due to the fact that we did not take into account the friction between the components when we performed the theoretical analysis of the human-machine system, nor did we consider the friction and contact force between the human and the machine and the friction and contact force between the human-machine system and the ground. As a result, the theoretical analysis model was too idealistic. Moreover, the simulation process of the structure may also have caused deviations from the theoretical analysis.

From the above simulation, the position of the centroid of the body model torso in the human-machine system could be obtained, and the speed-time curve of the deceleration module of each joint motor of the exoskeleton robot was obtained, as shown in Figures 13 and 14.

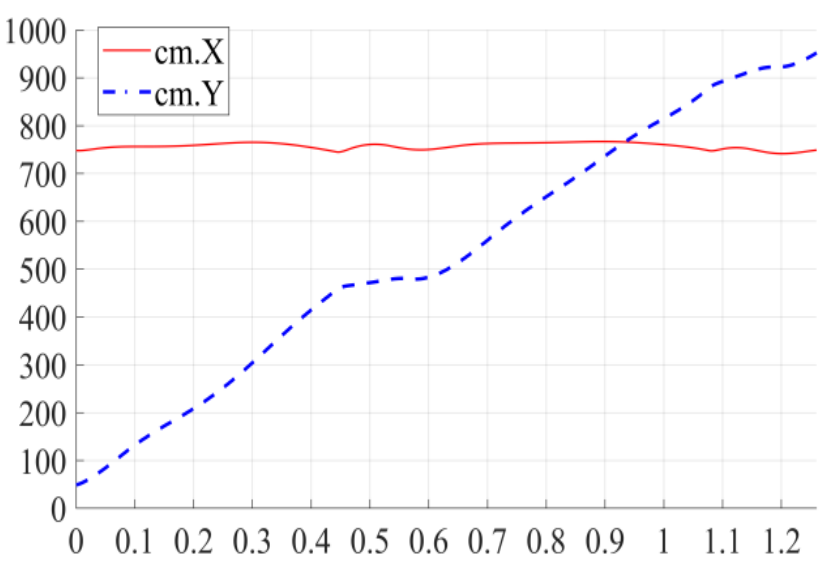

Figure 13. Position curve of the torso center of the mass of the human body model in the sagittal plane. 


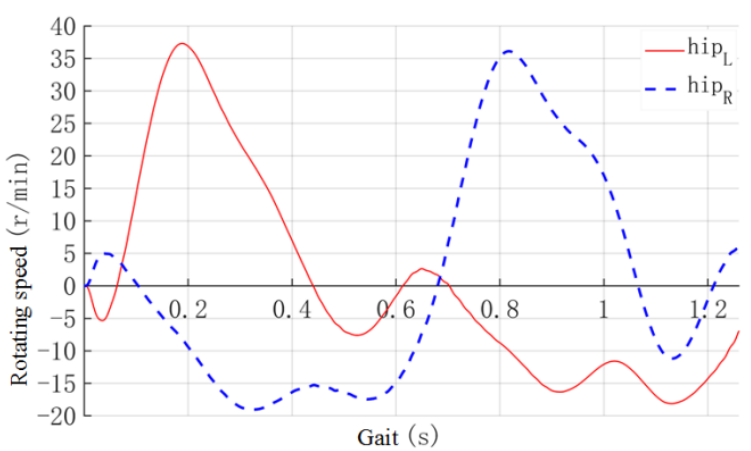

(a) Hip

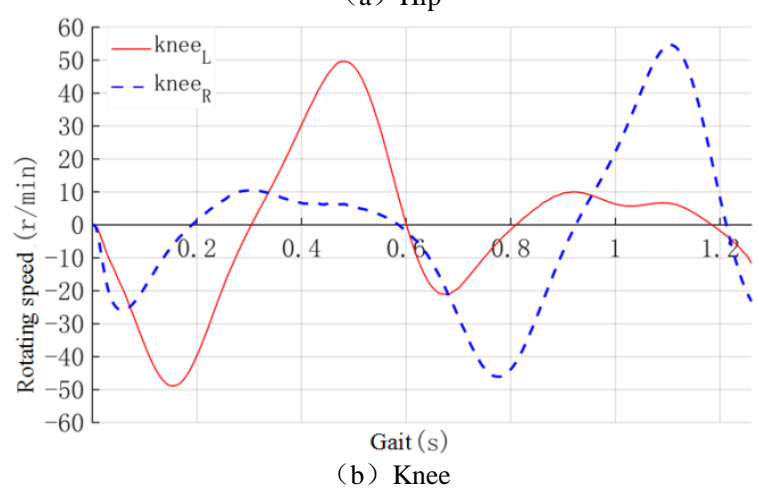

Figure 14. Speed-time curve of the deceleration module for each joint motor.

As can be seen from Figure 13, in the motion simulation process, the movement of the centroid of the human model torso in the sagittal plane was mainly manifested as follows: along the $x$ axis direction (that is, the vertical direction) up and down fluctuations occurred; along the $y$ axis in the positive direction (that is, the forward direction) displacement monotonically increased and, after calculations, its average speed was basically consistent with the actual movement of the human body. Therefore, the correctness of the simulation results was proved. From the simulation results of Figure 14, the maximum output speed requirement of the deceleration module of the external motors of the exoskeleton robot could be obtained. These results can be used for the specific selection of the deceleration module of each joint motor of the exoskeleton robot, and provide important theoretical parameters for physical prototype fabrication and control system design of lower extremity exoskeleton robots.

\section{Discussion and Conclusions}

This paper introduced a lower extremity exoskeleton robot, for which the degrees of freedom of each joint and their motion ranges were reasonably configured, and the mechanical structure of the exoskeleton robot was designed and modeled. The study also introduced the mechanical structure of the knee joint mechanism and its motion principle. The kinematic model of the proposed human-machine system was established by the D-H coordinate method, and the kinematic relationship between the knee joint mechanism and the human-machine system was established. By using MATLAB, the motion range of the ankle joint in the spatial sagittal plane-conforming to the actual motion situation - was solved, proving the correctness of the kinematic model established in this paper. The dynamic model of the human-machine system at different points in normal gait was established by the Lagrange method, and the torque of each joint of the human-machine system was determined. Then, the dynamic relationship between the knee joint mechanism and the human-machine system was established. The kinematics and dynamics simulations of the human-machine system model were carried out by ADAMS. The post-processing results of the simulations were basically consistent with the results calculated by importing the theoretical analysis into MATLAB, thus proving the correctness of the dynamic model established in this paper. At the same time, the position of the centroid of the human model torso in the sagittal plane was found to be basically in agreement with the actual motion during the simulation, proving the correctness of the simulation results. The relationship between the output speed and time of each joint motor deceleration module of the exoskeleton robot was solved by post-processing, providing important theoretical parameters for physical prototype fabrication and control system design of lower extremity exoskeleton robots.

There are, however, many other areas that remain to be studied: (1) the exoskeleton robot should be studied and analyzed in different terrain environments, with different motion speeds and different gait movement gaits; (2) the exoskeleton robot control system may be designed in greater detail to realize its motion control; and (3) further experiments and theoretical comparative analyses are needed in combination with physical prototype experiments.

\section{REFERENCES}

[1] Chen Ping, quiet. Conception of passive exoskeleton in patients [J]. Mechanical design \& amp; manufacturing, 2012 (6): 279-281.

[2] Dingmin, Li Jianmin, Wu Qingwen, et. Lower extremity gait rehabilitation robot: research progress and clinical application [J]. China Tissue Engineering Research, 2010, 14 (35): 6604-6607.

[3] Zoss A B, Kazerooni H, Chu A. Biomechanical design of the Berkeley lower extremity exoskeleton (BLEEX)[J]. IEEE/ASME Transactions on Mechatronics, 2006, 11(2):128-138.

[4] Gregorczyk K N, Hasselquist L, Schiffman J M, et al. Effects of a lower-body exoskeleton device on metabolic cost and gait biomechanics during load carriage[J]. Ergonomics, 2010, 53(10):1263-1275.

[5] Scarvell J, Scarvell J M, Smith K N, et al. Development of the concepts of knee kinematics [J]. Archives of Physical Medicine \& Rehabilitation, 2003, 84(12):1895-1902.

[6] Dong J H, Park H, Ha T, et al. Biomechanical design of an agile, electricity-powered lower-limb exoskeleton for weight-bearing assistance [J]. Robotics \& Autonomous Systems, 2017, 95:181-195.

[7] Clinical Gait Analysis. http://www.clinicalgaitanalysis.com/[OL].

[8] Stansfield B W, Hillman S J, Hazlewood M E, et al. Sagittal Joint Kinematics, Moments, and Powers Are Predominantly Characterized by Speed of Progression, Not Age, in Normal Children[J]. Journal of Pediatric Orthopaedics, 2001, 21(3):403-411.

[9] Siciliano, B,Khatib, O. Robot Manual [M]. Machinery industry Press, 2013:20-22.

[10] Denavit J. A kinematic notation for lower-pair mechanisms based on matrices [J]. Trans. of the ASME. Journal of Applied Mechanics, 1955, $22: 215-221$

[11] Sciavicco L, Siciliano B. Modeling and Control of Robot Manipu-lators [J]. Industrial Robot An International Journal, 1996, 21(1):99-100.

[12] Zoss A, Kazerooni H, Chu A. On the mechanical design of the Berkeley Lower Extremity Exoskeleton (BLEEX)[C]// 2005 IEEE/RSJ International Conference on Intelligent Robots and Systems. IEEE, 2005.

[13] Kim H G, Park S, Han C. Design of a novel knee joint for an exoskeleton with good energy efficiency for load-carrying augmentation [J]. Journal of Mechanical Science \& Technology, 2014, 28(11):4361-4367. 